\title{
Curved Structures in Soft Materials Stabilized by the Flexoelectric Effect
}

\author{
Thermodynamically Stable Blue Phases
}

F. Castles, S. M. Morris, E. M. Terentjev, and H. J. Coles

Physical Review Letters 104, 157801 (2010), published 13 April 2010

DOI:10.1103/PhysRevLett.104.157801

\section{Recommended with a commentary by Jonathan V. Selinger, Kent State University}

In liquid crystals, the flexoelectric effect is a coupling between curvature of the director field and electrostatic polarization. This coupling was first discovered in 1969 by Robert Meyer, ${ }^{1}$ who illustrated it with the drawing shown in Fig. 1. In a uniform nematic phase (1a), molecules are aligned along an axis, called the director $\mathbf{n}$. They are equally likely to point in either direction along this axis, and hence have no polar order. However, if a splay is applied to a nematic phase of pearshaped molecules (1b), then the symmetry between $+\mathbf{n}$ and $-\mathbf{n}$ is broken and the material acquires polar order, which can be observed as a macroscopic dipole moment

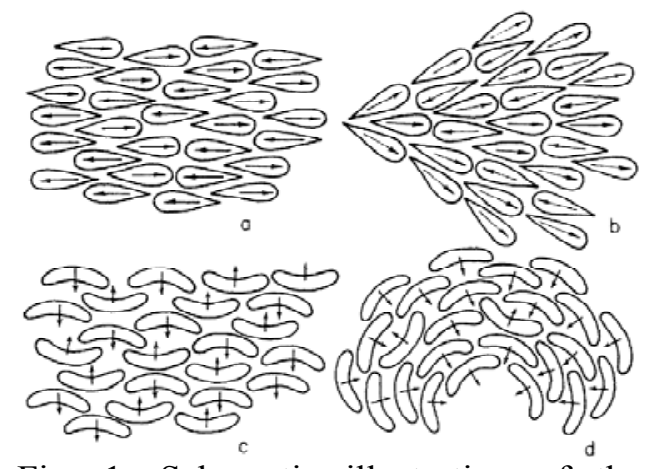

Fig. 1: Schematic illustration of the flexoelectric effect, from Meyer. ${ }^{1}$ along $\mathbf{n}$. Likewise, if a bend is applied to a nematic phase of banana-shaped or bent-core molecules (1c and d), the symmetry of rotations around $\mathbf{n}$ is broken and the material acquires polar order, now a macroscopic dipole moment transverse to $\mathbf{n}$. The same effects also work in reverse: An applied electric field induces an electrostatic polarization, which leads to splay or bend in the nematic director. Most liquid crystals have a combination of these two couplings, called the splay and bend flexoelectric effects, respectively.

Over the years, there have been many experimental measurements of the flexoelectric coefficients in different liquid-crystal materials, as well as many theoretical calculations of these coefficients based on the structure and statistical mechanics of liquid crystals. For typical liquid crystals, these coefficients are in the range of 3-20 pC/m. However, in recent experiments, Antal Jákli and collaborators ${ }^{2-4}$ have measured the flexoelectric coefficients of bent-core liquid crystals (with molecular structures similar to the schematic drawing in Figs. 1c and d), and they find a bend flexoelectric coefficient of up to $35 \mathrm{nC} / \mathrm{m}$, roughly three orders of magnitude larger than the typical value. With this large coupling, bent-core liquid crystals may be practical materials for converting mechanical into electrical energy.

Although the flexoelectric effect is normally detected using the electrostatic polarization, and although its applications may involve electrical energy, we should note that the flexoelectric effect is not inherently related to electricity. Rather, one should just think of this effect as a coupling between polar order and curvature of the director. The electric dipole moment is only a marker for polar order, which allows the order to be detected macroscopically. The same ordering would occur even if the molecules did not have any electric dipole moment. Hence, one should expect the flexoelectric effect to be important whenever liquid crystals or other soft materials have highly curved configurations of the molecular orientation.

This perspective on the flexoelectric effect brings us to the new article on "Thermodynamically Stable Blue Phases," by Harry Coles, Eugene Terentjev, and their 
collaborators at the University of Cambridge. ${ }^{5}$ In this article, they propose that flexoelectricity plays an important role in stabilizing blue phases of chiral liquid crystals. Blue phases are complex structures consisting of double-twist cylinders, as shown in Fig. 2. As explained by Meiboom et al., ${ }^{6,7}$ each double-twist cylinder has the optimal local packing of molecules, but this local packing cannot extend to fill space globally, a phenomenon called geometric frustration. ${ }^{8}$ Hence, the

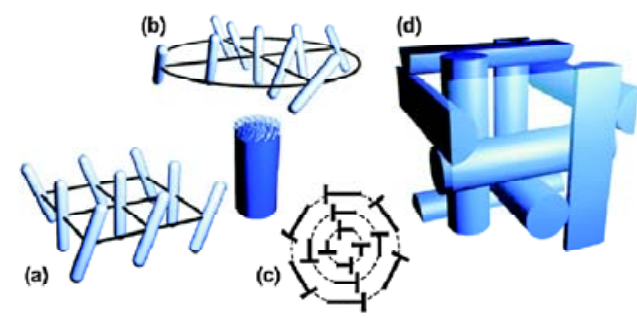

Fig. 2: Double-twist cylinders in a blue phase, from Castles et al. ${ }^{5}$

blue phases form as double-twist cylinders separated by defect lines. For many years, blue phases were considered as a very esoteric branch of liquid-crystal science, not connected with applications. However, recent research has developed a promising new electro-optic display technology based on blue phases. ${ }^{9}$ This revival of blue-phase research has been discussed in a Condensed Matter Journal Club commentary by Slobodan Zumer. ${ }^{10}$

In typical liquid crystals, blue phases form over a very narrow temperature range of about $1^{\circ} \mathrm{C}$. Motivated by the new electro-optic applications, Coles and collaborators have developed new liquid crystals with blue phases over a much wider range of about $50^{\circ} \mathrm{C}$, including room temperature. ${ }^{11}$ These materials have a bent-core structure, similar to the liquid crystals discussed for the flexoelectric effect above. This experimental result leads to the theoretical question: What stabilizes the blue phases over such a wide temperature range in these liquid crystals?

The new article argues that this stabilization occurs precisely because of the large flexoelectric effect. In intuitive terms, one might say that the blue phase requires a high curvature of the director field, which can occur easily in bent-core liquid crystals, as in the classic illustration of Fig. 1d. The only free-energy cost would be the entropic cost of aligning the dipole moments (and this entropic cost might be partially offset by an energetic benefit ${ }^{12}$ ). In more mathematical terms, the authors present a continuum elastic model of the free energy of liquid crystals, which includes the flexoelectric coupling between polar order and curvature of the director. They then integrate out the polar order to obtain an effective free energy in terms of the director alone. In this free energy, the elastic constants for splay and bend distortions are renormalized (or reduced) by an amount proportional to the squares of the splay and bend flexoelectric coefficients, respectively. With reasonable estimates for the relevant material parameters, this renormalization leads to an increase in the temperature range of the blue phase, consistent with experiments. Thus, the model for flexoelectricity accounts for the change in bluephase properties, and shows how further liquid crystals can be designed for blue-phase applications.

If blue phases can be stabilized by the flexoelectric effect, then a logical next question is: What else can be stabilized by the flexoelectric effect? Blue phases are not the only highly curved structures in soft materials; we might expect that the same flexoelectric mechanism would affect other structures as well. Paolo Biscari and Maria Carme Calderer have investigated a similar concept in the context of smectic liquid-crystal fibers; they find that a coupling between curvature and polar order leads to a "telephone-cord" helical instability in the shape of the fibers. ${ }^{13}$ My former postdoc Zhao Lu and I have used the flexoelectric effect to model bilayer membranes composed of diacetylenic lipids. These molecules have kinked shapes similar to bent-core liquid crystals, and the membranes tend to curl into cylindrical tubules. This curvature was previously attributed to molecular chirality, ${ }^{14}$ but we now argue that it might be better explained by flexoelectricity. ${ }^{15}$ 
Most recently, Elizabeth Mann and collaborators have used Brewster-angle microscopy to image patterns of molecular tilt orientation in Langmuir monolayers of diacetylenic lipids on the surface of water. ${ }^{16}$ Their experiments find remarkably complex modulated structures,
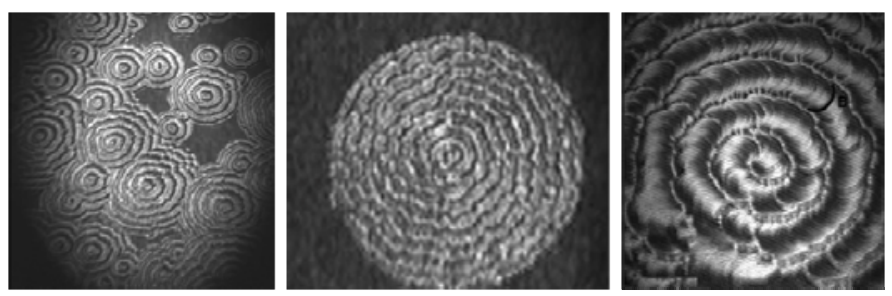
as shown in Fig. 3. Although no detailed in lipid monolayers, from Mann et al. ${ }^{16}$ theory of these structures has yet been proposed, we speculate that they might also arise from the flexoelectric effect, which could couple polar order in the molecular kinks with bend in the director field. In that view, these structures could be two-dimensional analogues of liquid-crystal blue phases, showing the generality of the flexoelectric mechanism for stabilizing curved structures.

${ }^{1}$ R. B. Meyer, "Piezoelectric Effects in Liquid Crystals," Physical Review Letters 22, 918-921 (1969).

2 J. Harden, B. Mbanga, N. Éber, K. Fodor-Csorba, S. Sprunt, J. T. Gleeson, and A. Jákli, "Giant Flexoelectricity of Bent-Core Nematic Liquid Crystals," Physical Review Letters 97, 157802 (2006).

${ }^{3}$ J. Harden, R. Teeling, J. T. Gleeson, S. Sprunt, and A. Jákli, "Converse flexoelectric effect in a bentcore nematic liquid crystal," Physical Review E 78, 031702 (2008).

${ }^{4}$ J. Harden, M. Chambers, R. Verduzco, P. Luchette, J. T. Gleeson, S. Sprunt, and A. Jákli, "Giant flexoelectricity in bent-core nematic liquid crystal elastomers," Applied Physics Letters 96, 102907 (2010).

${ }^{5}$ F. Castles, S. M. Morris, E. M. Terentjev, and H. J. Coles, "Thermodynamically Stable Blue Phases," Physical Review Letters 104, 157801 (2010).

${ }^{6}$ S. Meiboom, P. W. Anderson, and W. F. Brinkman, "Theory of the Blue Phase of Cholesteric Liquid Crystals," Physical Review Letters 46, 1216-1219 (1981).

${ }^{7}$ S. Meiboom, M. Sammon, and W. Brinkman, "Lattice of disclinations: The structure of the blue phases of cholesteric liquid crystals," Physical Review A 27, 438-454 (1983).

${ }^{8}$ For a review of geometric frustration in liquid crystals, see R. D. Kamien and J. V. Selinger, "Order and frustration in chiral liquid crystals," Journal of Physics: Condensed Matter 13, R1-R22 (2001).

${ }^{9}$ H.-S. Kitzerow, "Blue Phases at Work!," ChemPhysChem 7, 63-66 (2006).

${ }^{10} \mathrm{~S}$. Zumer, "Blue phase revival," http://www.condmatjournalclub.org/?p=1055 (2010).

${ }^{11}$ H. J. Coles and M. N. Pivnenko, "Liquid crystal 'blue phases' with a wide temperature range," Nature 436, 997-1000 (2005).

${ }^{12}$ For liquid crystals on the verge of a polar phase, see S. Dhakal and J. V. Selinger, "Statistical mechanics of splay flexoelectricity in nematic liquid crystals," Physical Review E 81, 031704 (2010), further article on bend in preparation.

${ }^{13}$ P. Biscari and M. C. Calderer, "Telephone-cord instabilities in thin smectic capillaries," Physical Review E 71, 051701 (2005).

${ }^{14}$ J. V. Selinger, M. S. Spector, and J. M. Schnur, "Theory of Self-Assembled Tubules and Helical Ribbons," Journal of Physical Chemistry B 105, 7157-7169 (2001).

${ }^{15}$ J. V. Selinger and Z. Lu, "Shape selection in self-assembled chiral membranes: New mechanism based on the flexoelectric effect," presentation at 21st International Liquid Crystal Conference, http://ilcc.confex.com/ilcc/2006/techprogram/P2376.HTM (2006), article in preparation.

${ }^{16}$ P. B. Basnet, E. K. Mann, and S. Chaieb, "Pattern Formations in Langmuir Films of Chiral Lipids," presentation at 84th Colloid and Surface Science Symposium (2010), article in preparation. 